\title{
The Research of R\&D Spillover and Total Factor Productivity of China
}

\author{
Wu Ming-e ${ }^{1,2}$ \\ ${ }^{1}$ College of Public Administration, Chongqing University, Chongqing, China \\ ${ }^{2}$ College of Economics, Southwest University of Political Science and Law, Chongqing, China
}

\section{Email address:}

wuminge502@126.com

\section{To cite this article:}

Wu Ming-e. The Research of R\&D Spillover and Total Factor Productivity of China. Science Innovation. Vol. 5, No. 2, 2017, pp. $102-107$. doi: 10.11648/j.si.20170502.16

Received: March 17, 2017; Accepted: April 6, 2017; Published: April 12, 2017

\begin{abstract}
By estimating the index of capital services which has double characteristics of material quantity and price with perpetual inventory method and the index of labor input which can indicate quality change with Iterative Proportional Fitting method, we recalculate China's total factor productivity and sources of economic growth from 1978 to 2010 by using growth accounting. Then we test the correlation between R\&D spillover and TFP of China on two ways of international trade and FDI. The results show that the improvement of TFP in China depends on the comprehensive benefit of domestic R\&D input and international R\&D spillover. Independent R\&D input is the dominant factor of China's TFP ascension. R\&D spillover from international trade and FDI has a time-lag effect on promoting China's TFP, while has a cause-and-effect relationship in the long term.
\end{abstract}

Keywords: R\&D Spillover, TFP, International Trade, FDI

\section{R\&D溢出与中国全要素生产率的研究}

\author{
吴明娥 ${ }^{1,2}$ \\ 1公共管理学院, 重庆大学, 重庆, 中国 \\ ${ }^{2}$ 经济学院, 西南政法大学, 重庆, 中国
}

邮箱

wuminge502@126. com

摘要：基于永续盘存法估算出具有物量和价格双重属性的资本服务物量指数，采用迭代比例拟合算法将就业人数及其 劳动报酬按行业、性别、年龄、文化程度进行四维交叉分类测算出中国劳动投入指数, 在增长核算框架下对中国全要 素生产率 (TFP) 进行更为谨慎的再测算。在此基础上, 从国际贸易和FDI双重溢出视角实证检验R\&D溢出与中国TFP增 长的相关关系。经验分析表明：中国TFP的提高取决于国内自主R\&D投入和国际R\&D溢出的综合效益，其中自主R\&D投入 是中国TFP提升的主导因素; 国际贸易和FDI途径下的R\&D溢出对中国TFP的推动作用具有时滞效应, 长期内呈双向因果 关系。

关键词: R\&D溢出, 全要素生产率, 国际贸易, FDI 


\section{1. 引言}

改革开放以来, 中国经济实现了举世瞩目的高速增长, 国际贸易量和外商直接投资额不断增加, 并于 2010 年超过 日本成为世界第二大经济体。新增长理论认为, 创新活动 是一国技术进步和生产力增长的重要引擎 (Romer, 1990), 而创新主要来自于研究与开发 (R\&D) 活动的积累。因此, 诸多文献针对国际间R\&D溢出的主要渠道——国际贸易和 外商直接投资 (FDI) 与全要素生产率 (TFP) 的关系进行 了广泛探讨。Grossman和Helpman (1991)、Coe和Helpman (1995) 、Bin Xu和Jianmao Wang (1999) 、黄先海和石 东楠 (2005)、李小平和朱钟棣 (2006)、郭峰等 (2013)、 高静和黄繁华 (2013) 等研究均表明, 国际贸易过程中伴 随着显著的技术溢出效应, 并与进口国的生产率增长显著 正相关。与国际贸易相比, 学者对TFP是否受益于通过FDI 渠道溢出的R\&D资本所持观点不尽相同。Barrel1和Pain （1999）、Sadik和Bolbol (2001)、沈坤荣和耿强 (2001)、 潘文卿（2003）、谢建国（2006）等研究表明FDI进入会 带来显著的技术溢出效应, 对生产率的提高和技术进步起 到了积极作用; 而Aitken和Harrison（1999）、Veugelers 和Cassiman（2004）、张海洋和刘海云（2004）、王春法 等 (2004) 、赵奇伟和张诚（2006）、元朋等（2008）、 马林和章凯栋（2008）、陈琳和林珏（2009）、张中元和 赵国庆 (2012) 等研究发现外资进入对当地企业或行业进 行技术转移的 “溢出效应” 非常有限, 有的甚至呈负的溢 出效应。

纵观现有文献, 学者们从理论和实证两方面不断完善 R\&D溢出与全要素生产率的相关性研究, 取得了丰富成果, 但仍存在值得进一步探讨之处, 主要表现在: (1)模型构建 上, 现有研究大多集中在国际R\&D溢出的单一路径分析上, 或者将国际贸易和FDI合并成为综合的对外开放指标来研 究 (如Coe和Helpman, 1995; Edwards, 1998; Wu, 2004; 许和连等, 2006; Jiang, 2011; 毛其淋和盛斌，2011）， 而未将国际贸易和FDI两种R\&D溢出渠道纳入到统一的分 析框架之中, 从而无法辨析国际贸易和FDI在R\&D溢出效应 上的不同表现。(2)指标选取上, 绝大多数文献在测度TFP 时, 对资本投入和劳动投入数据缺少最低限度的关注。在 新古典生产理论中, 资本是即时生产能力的概念, 其在生 产过程中所提供的是服务流而非存量; 而劳动投入也应当 是一定时期内劳动力提供的 “服务流量” , 它不仅取决于 劳动力数量, 而且还与劳动力的利用效率、质量等因素有 关。因此, 采用资本存量和就业人数来分别衡量资本投入 和劳动投入的通常做法存在一定缺陷, 从而可能给TFP的 测算带来误差。

基于此, 在充分考虑现有研究成果的基础上, 本文试 图从以下几个方面对现有文献进行拓展：(1)鉴于国际R\&D 溢出途径主要包括国际贸易和FDI, 本文将基于LP模型 （Lichtenberg和Potterie，1998）分别测算国际贸易和 FDI途径溢出的国际R\&D资本, 并将二者纳入到统一的实证 分析框架之中, 以期更为全面地考察国际R\&D溢出对中国 TFP的影响。(2)以资本服务而非资本存量作为资本投入的 替代度量, 而在资本服务的测算上, 与现有研究对不同资 产采用综合折旧率不同，本文利用 “年龄一效率” 函数和
“年龄一价格” 函数的相关关系，运用资产价值公式获得 历年资本存量净额和折旧额, 估算出不同资产历年折旧率 的时间序列数据, 并使用统一口径的官方最新统计数据, 借鉴澳大利亚统计局（ABS）、美国劳工统计局（BLS）以 及加拿大统计局 (SC) 的做法, 明确和规范中国总量层次 的资本服务估算步骤及细节处理，从而避免不同方法对估 算结果准确性的影响。(3)考虑到以就业人数作为劳动投入 的衡量方式不能反映劳动时间和劳动质量变化, 本文将就 业人数及其劳动报酬按 20 个行业、男女性别、7种文化程 度、 11 个年龄段进行四维交叉分类, 与以往研究相比, 对 劳动投入的文化程度和年龄层次进行更为细致的划分, 并 利用历年《中国劳动统计年鉴》等统计资料构建 1985 年以 来历年就业人数和劳动报酬全维矩阵, 保证数据统计口径 的一致性和连续性, 运用迭代比例拟合算法 (IPF) 对中 国劳动投入进行谨慎测度。

\section{2. 模型设定与R\&D溢出测算}

\section{1 . 模型设定}

为了分析国际贸易和FDI途径下的R\&D溢出与TFP的关 系, 本文引入传统的 $\mathrm{C}-\mathrm{D}$ 总生产函数:

$$
Y=A K^{\alpha} L^{\beta} S^{\gamma}
$$

其中， $Y$ 表示国民生产总值， $A$ 表示技术进步， $K$ 表 示物质资本投入, $L$ 表示劳动投入, $S$ 表示知识资本投入。 在增长核算框架下，TFP表示不能由要素投入增长所解释 的产出增长部分, 即可将其定义为总产量与要素投入量之 比:

$$
T F P=Y / K^{\alpha} L^{\beta}=A S^{\gamma}
$$

根据Coe和Helpman (1995) 提出的贸易溢出计量模型 （简称 $\mathrm{CH}$ 模型），在开放经济条件下，一国的知识资本 $S$ 取决于本国R\&D资本投入以及国外R\&D资本溢出, 而国外 R\&D资本溢出主要来源于国际贸易和FDI。因此, 可将TFP 进一步表示为:

$$
T F P=A S(O)^{\alpha} S(I T)^{\beta} S(F D I)^{\gamma}
$$

其中, $S(O)$ 为本国 $\mathrm{R} \& D$ 资本投入, $S(I T)$ 和 $S(F D I)$ 分 别表示通过国际贸易和FDI途径溢出的R\&D资本。将式 (3) 两边取对数, 则可得到如下基本回归方程:

$$
\begin{aligned}
\ln T F P= & \ln A+\alpha \ln S(O)+\beta \ln S(I T) \\
& +\gamma \ln S(F D I)+\varepsilon
\end{aligned}
$$

其中, $\varepsilon$ 为随机扰动项。通过对式 (4) 进行计量经 济分析, 即可看出国际贸易和FDI途径下R\&D溢出与TFP之 间的关系。

\section{2. R\&D溢出测算}

Coe和Helpman（1995）开创性地使用进口份额作为权 重构造国外R\&D资本，建立CH模型后，Lichtenberg和 
Potterie（1998）对CH模型的设定偏差进行了修正, 提出 了另一种测度方法（简称LP）, 即：

$$
S(I T)=\sum_{j \neq i} \frac{S_{j t}^{d}}{Y_{j t}} M_{i j t}
$$

其中, $S(I T)$ 表示 $t$ 时期通过国际贸易途径溢出到 $i$ 国 的国际R\&D资本存量, $S_{j t}^{d}$ 表示 $t$ 时期 $j$ 国国内R\&D资本存量, $Y_{j t}$ 表示 $t$ 时期 $j$ 国的GDP, $M_{i j t}$ 表示 $t$ 时期 $i$ 国从 $j$ 国的进 口额。本文采用此测度方法, 选择1985-2010中国累计进 口前十位国家作为中国主要贸易对象, 对中国国际贸易途 径的国际R\&D资本存量进行测算 $[1]$ 。参照历年《中国统计 年鉴》，中国主要贸易对象依次为: 日本、韩国、美国、 德国、澳大利亚、新加坡、法国、加拿大、意大利和英国。 中国从各国的进口数据来自历年《中国统计年鉴》, 各个 国家的R\&D资本存量占GDP比重数据来自历年《中国科技统 计年鉴》。由于部分年份数据缺失, 本文采用直线插值法 对缺失年份数据进行补充。

为保证测量方法的一致性, 本文同样使用LP方法测度 FDI途径下的国外R\&D溢出, 其公式为:

$$
S(F D I)=\sum_{j \neq i} \frac{S_{j t}^{d}}{Y_{j t}} F D I_{i j t}
$$

其中, $S(F D I)$ 表示 $t$ 时期通过FDI途径溢出到 $i$ 国的 R\&D资本存量, $S_{j t}^{d}$ 和 $Y_{j t}$ 变量含义同式 $(5), F D I_{i j t}$ 表示 $t$ 时期 $j$ 国流入 $i$ 国的实际外商直接投资额。本文选择 1985-2010累计对中国实际直接投资额前十位国家作为中 国主要外资来源, 对中国FDI途径下的国际R\&D资本存量进 行测算。参照历年《中国统计年鉴》，中国外商直接投资 额主要来源于以下国家: 日本、美国、韩国、新加坡、德 国、英国、法国、加拿大、澳大利亚和意大利。

\section{3. 全要素生产率测算}

由于可以对TFP进行再分解且不需要对生产函数和误 差项进行设定, 现有文献倾向采用以数据包络分析 (DEA) 为代表的非参数法来测算TFP。非参数法对数据质量和连 续性的要求非常高, 受统计口径影响, 中国产出数据、就 业人数以及基于资本形成或全社会固定资产投资估算的 资本投入数据都是严重不连续的 [2], 从而可能给以DEA 为代表的非参数法带来严重系统误差。因此, 本文选用增 长核算法测度TFP。

\section{1 . 资本服务测算}

由于无法直接测量资本服务, 资本服务的测算是以资 本存量的估算为基础。本文估算资本服务的处理步骤为: 首先，采用1985-2010年中国固定资本形成序列和固定资 本投资序列数据, 运用永续盘存法 (PIM) 估算资本存量 总额; 其次, 分别利用双曲线形态下的 “年龄一效率” 函 数和 “年龄一价格” 函数以及二者的相关关系估算生产性
资本存量; 最后, 以通过内生方法得到的资本租赁价格作 为权数, 用Tornqvist指数形式对不同类型资产进行加总, 得出具有物量和价格双重属性的资本服务物量指数。与以 往研究不同之处在于, 鉴于折旧率对估算结果的重要影响, 本文首先采用双曲相对效率模式, 利用资产正态退出函数 和残存函数, 通过截断处理准确地描述资产效率损失和磨 损; 其次利用 “年龄一效率” 函数和 “年龄一价格” 函数 之间的关系，通过资产价值公式推导出 “年龄一价格” 函 数, 由此得到资产历年的折旧额和资本存量净额, 二者相 比即可求出不同类型资产历年折旧率的时间序列数据。具 体测算过程可参见课题组前期研究 [3-5], 此处不再赘述。

\section{2. 劳动投入测算}

\section{2. 1. 估算方法}

根据Jorgenson提供的方法论 [6], 经济活动中劳动投 入总量可以表示为具有不同特征的劳动投入分量的函数, 函数形式一般采用超越对数函数, 则劳动投入可以表示为:

$$
\ln L=\alpha_{0}+\sum_{i=1}^{n} \alpha_{i} \ln L_{i}+\frac{1}{2} \sum_{i=1}^{n} \sum_{j=1}^{n} \beta_{i j} \ln L_{i} \times \ln L_{j}
$$

其中, $i=1,2, \ldots, n, j=1,2, \ldots, n, \alpha_{0} 、 \alpha_{i} 、 \beta_{i j}$ 为 估计参数, $L_{i} 、 L_{j}$ 分别表示 $i$ 类和 $j$ 类劳动投入分量。在 超越对数函数形式下, 度量劳动投入变化的公式可表示为:

$$
\begin{aligned}
g_{t} & =\ln L_{t}-\ln L_{t-1}=\sum_{i=1}^{n} \bar{V}_{i, t}\left[\ln L_{i, t}-\ln L_{i, t-1}\right] \\
& =\sum_{i}^{n} \bar{V}_{i, t} \times g_{t, t-1}^{i}
\end{aligned}
$$

其中, $L_{t} 、 L_{t-1}$ 分别表示第 $t$ 期、 $t-1$ 期的劳动投入 总量, $g_{t, t-1}^{i}$ 表示第 $i$ 类劳动投入分量在 $t$ 期和 $t-1$ 期间的 增长速度, 权数 $\bar{V}_{i, t}$ 为 $t$ 期和 $t-1$ 期相应价值份额的均值:

$$
\bar{V}_{i, t}=\frac{1}{2}\left[V_{i, t}+V_{i, t-1}\right]
$$

而价值份额可以表示为:

$$
V_{i, t}=\frac{w_{i, t}}{W_{t}}
$$

其中, $w_{i, t}$ 为第 $t$ 期第 $i$ 类劳动投入分量的价格 (劳动 报酬）， $W_{t}$ 为第 $t$ 期劳动投入总量的价格（劳动报酬）。 由式 (8) 可以看出, 劳动投入变化指数等于不同种类劳 动投入分量增长速度的加权和, 权数为各种劳动投入分量 劳动报酬比重在 $t$ 期和 $t-1$ 期之间的均值。

\subsection{2. 数据处理}

为了区别不同类型就业人员的劳动质量, 以往研究将 就业人员按行业、性别、年龄、受教育程度、职业、就业 身份等属性进行交叉分类, 各类就业人员不同的平均劳动 
报酬反映了不同就业人员的素质。因此, 测量劳动投入指 数的关键是要具备多维交叉分组的就业人数及其劳动报 酬的详细分类数据, 但根据中国现有公开的统计资料很难 直接获得上述交叉分组数据。为了弥补现有统计资料缺陷, 本文参照任若恩、岳希明和郑海涛等 (2013) 的研究, 采 用迭代比例拟合算法 (IPF) 对现有数据进行处理 [7]。IPF 法最初由Bishop（1975）提出, 其基本原理为假定一个全 维矩阵 (所需要的维数较多的矩阵), 通过迭代法不断对 初始数据进行调整, 使全维矩阵的元素依次满足边际矩阵 (维数少于全维的矩阵) 给出的控制总量, 直到元素收玫 达到期望的精度。

以往研究在采用哪些就业人员属性进行分组以及属 性分组的详细程度上各有不同, 这在很大程度上取决于数 据的可利用程度 [8]。基于现有数据的可利用程度, 本文 将就业人员按 20 个行业、男女性别、7种文化程度、11个 年龄段进行四维交叉分类, 目标在于建立1985-2010年关 于就业人数和劳动报酬的四维交叉分类矩阵的时间序列, 表1给出了详细的分组信息。

为了能够详细测算中国每年的劳动投入变化情况, 本 文未使用 10 年一次的人口普查和中间年份的 $1 \%$ 人口抽样 调查数据, 而是通过历年《中国劳动统计年鉴》得到了按 行业、性别、年龄分类的，按性别、年龄、文化程度分类 的以及按行业、性别、文化程度分类的三个三维就业人数 边际矩阵, 分别用 $N_{t s a} 、 N_{s a e} 、 N_{t s e}$ 表示, 通过使用 IPF 法迭代至收敛得到全维就业人数矩阵 $N_{t s a e}$ ( $t 、 s 、 a 、 e$ 分别表示行业、性别、年龄和文化程度）。

表1 就业人员分组信息。

\begin{tabular}{|c|c|c|c|}
\hline 行业 & & & \\
\hline 序号 & 行业分组 & 序号 & 行业分组 \\
\hline 1 & 农、林、牧、渔业 & 11 & 房地产业 \\
\hline 2 & 采矿业 & 12 & 租赁和商务服务业 \\
\hline 3 & 制造业 & 13 & $\begin{array}{l}\text { 科学研究、技术服务和地质 } \\
\text { 勘查业 }\end{array}$ \\
\hline 4 & $\begin{array}{l}\text { 电力,燃气及水的 } \\
\text { 生产和供应业 }\end{array}$ & 14 & $\begin{array}{l}\text { 水利、环境和公共设施管理 } \\
\text { 业 }\end{array}$ \\
\hline 5 & 建筑业 & 15 & 居民服务和其他服务业 \\
\hline 6 & $\begin{array}{l}\text { 交通运输、仓储和 } \\
\text { 邮政业 }\end{array}$ & 16 & 教育 \\
\hline 7 & $\begin{array}{l}\text { 信息传输、计算机 } \\
\text { 服务和软件业 }\end{array}$ & 17 & $\begin{array}{l}\text { 卫生、社会保障和社会福利 } \\
\text { 业 }\end{array}$ \\
\hline 8 & 批发和零售业 & 18 & 文化、体育和娱乐业 \\
\hline 9 & 住宿和餐饮业 & 19 & 公共管理和社会组织 \\
\hline $\begin{array}{l}10 \\
\text { 年龄 }\end{array}$ & 金融业 & $\begin{array}{l}20 \\
\text { 性别 }\end{array}$ & 国际组织 \\
\hline 序号 & 年龄分组 & 序号 & 性别分组 \\
\hline 1 & $16-19$ & 1 & 男 \\
\hline 2 & $20-24$ & 2 & 女 \\
\hline 3 & $25-29$ & 文化程度 & \\
\hline 4 & $30-34$ & 序号 & 文化程度分组 \\
\hline 5 & $35-39$ & 1 & 未上过学 \\
\hline 6 & $40-44$ & 2 & 小学 \\
\hline 7 & $45-49$ & 3 & 初中 \\
\hline 8 & $50-54$ & 4 & 高中 \\
\hline 9 & $55-59$ & 5 & 大学专科 \\
\hline 10 & $60-64$ & 6 & 大学本科 \\
\hline 11 & $65+$ & 7 & 研究生 \\
\hline
\end{tabular}

编制劳动投入指数同时还需要与全维就业人数矩阵 完全相对应的全维劳动报酬矩阵。《中国劳动统计年鉴》 提供了中国历年分行业的就业人员平均工资矩阵，用 $W_{t}$ 表示, 为了剔除物价影响, 我们使用居民消费价格指数对 其进行平减。中国收入分配研究院联合国外研究者广泛调 查形成的中国居民收入调查数据库 (CHIPS) 为本文提供 了按性别、年龄、文化程度分类的就业人员年平均工资矩 阵, 用 $W_{s a e}$ 表示, 同样使用居民消费价格指数对其进行平 减。在得到全维就业人数矩阵 $N_{t s a e}$ 和两个平均工资边际矩 阵 $W_{t} 、 W_{s a e}$ 的情况下, 使用IPF法迭代至收玫即可估算出 历年全维劳动报酬矩阵 $W_{\text {tsae }}$ 。在此基础上, 利用式 (8) 即可求解得到1985-2010年中国劳动投入指数的编制结果。

\section{3. 产出与要素产出弹性测算}

为了与资本投入估算数据保持统一口径与基期, 本文 采用GDP作为产出指标, 数据来源于历年《中国统计年鉴》, 并以1978年不变价格进行换算。在利用收入份额法确定要 素产出弹性时, 李宾和曾志雄 (2009) 采用分城镇和乡村 “人均收入乘以人口数” 获取总劳动收入的思路计算劳动 收入份额 [9], 从而避免了因统计口径调整造成的影响, 本文也借鉴该思路来测算资本和劳动投入的产出弹性。

\section{4. 实证检验与分析}

\section{1 . 单位根检验}

由于现实经济中大多数变量都是非平稳的, 从而可能 导致伪回归现象, 因此在对变量进行协整分析之前, 有必 要对各变量进行单位根检验, 确定各变量的单整性与单整 阶数。依据表 2 的ADF检验结果, 变量 $\ln T F P 、 \ln S(O)$ 、 $\ln S(I T)$ 和 $\ln S(F D I)$ 的水平序列都是非平稳的, 但它们的 一阶差分在 $5 \%$ 的显著性水平下是平稳的, 即均属于I (1) 序列。

表2 各变量的单位根检验。

\begin{tabular}{llllll}
\hline 变量 & ADF值 & $\begin{array}{l}\text { 滞后 } \\
\text { 阶数 }\end{array}$ & $\begin{array}{l}\text { 置信 } \\
\text { 概率 }\end{array}$ & $\begin{array}{c}\text { 临界值 } \\
(5 \%)\end{array}$ & $\begin{array}{l}\text { 检验 } \\
\text { 结果 }\end{array}$ \\
\hline $\ln T F P$ & -1.5167 & 1 & 0.7950 & -3.6122 & 不平稳 \\
$\ln S(O)$ & -2.8478 & 1 & 0.1966 & -3.6329 & 不平稳 \\
$\ln S(I T)$ & -2.7783 & 1 & 0.2173 & -3.6032 & 不平稳 \\
$\ln S(F D I)$ & -0.4648 & 1 & 0.9785 & -3.6032 & 不平稳 \\
$\Delta \ln T F P$ & -4.2458 & 1 & 0.0143 & -3.6220 & 平稳 \\
$\Delta \ln S(O)$ & -5.1131 & 1 & 0.0030 & -3.6585 & 平稳 \\
$\Delta \ln S(I T)$ & -2.2133 & 1 & 0.0286 & -1.9557 & 平稳 \\
$\Delta \ln S(F D I)$ & -2.9744 & 1 & 0.0047 & -1.6088 & 平稳 \\
\hline
\end{tabular}

\section{2. 协整检验}

由于变量的一阶差分序列均已平稳, 满足协整检验前 提。从检验手段上可以将协整检验分为两种: 一种是基于 回归残差的EG (Engle和Granger, 1987) 两步法协整检验, 另一种是在VAR系统下用极大似然估计来检验多变量间协 整关系的方法, 即 Johansen协整检验（Johansen 和 
Juselius，1990），本文采用后者进行分析。建立VAR模 型除了要满足平稳性条件外, 还需正确确定滞后期 $k$ 。如 果滞后期太少, 误差项的自相关会很严重, 并导致参数的 非一致性估计。本文根据LR统计量、AIC和SC信息准则来 确定最优滞后期, 经反复测试发现, AIC和SC统计量在滞 后 4 期时同时达到最小, 因此可以确定最优滞后期数为 4 。 我们利用White检验和JB检验进一步检验滞后期数为 4 的 VAR模型, 发现其拟合优度很好, 残差序列具有平稳性。 协整检验结果显示, $\ln T F P 、 \ln S(O) 、 \ln S(I T)$ 和 $\ln S(F D I)$ 在 $5 \%$ 的显著性水平上存在一个协整方程。这表 明, 上述四个变量至少在最优滞后期内存在着一个长期稳 定的均衡关系。根据向量误差修正模型我们得到标准化的 协整向量:

$$
\begin{aligned}
\ln T F P= & -4.2765+0.3862 \ln S(O)+0.0939 \ln S(I T) \\
& +0.0432 \ln S(F D I)
\end{aligned}
$$

可以看出, 国内自主R\&D投入和国外R\&D溢出对中国 TFP的提升均具有正向促进作用。国内自主R\&D投入、国际 贸易和FDI途径下的R\&D溢出每提高 1 个百分点, 可带动TFP 分别增长 $0.3862 、 0.0939$ 和 0.0432 个百分点。显然, 国内 自主R\&D投入是中国TFP提升的主导因素, 而国际贸易和 FDI途径下的R\&D溢出虽对中国TFP提升呈正向作用, 但作 用强度较小，其对提高中国TFP的作用并没有普遍认为的 那样显著。究其原因, 可能为: 第一, 为获取超额垄断利 润, 跨国公司采取技术封锁等措施限制先进技术外溢, 阻 碍中国企业获得其核心技术; 第二, 中国国际贸易呈现加 工贸易倾向, 进口产品集中在附加值低、技术含量低的劳 动密集型行业, 技术溢出效应不显著。而对于技术含量高 的加工贸易的进口, 又集中在存在技术黑箱无法模仿的关 键零部件上, 从而很大程度上阻碍了国内产业链的延长, 导致关键零部件和原材料过度依赖进口, 中间产品不能实 现进口替代 [10]; 第三, 中国企业先进技术吸收能力不足, 影响了国际贸易和FDI途径下R\&D溢出效果。

\section{3. 格兰杰因果关系检验}

由于 $\ln T F P 、 \ln S(O) 、 \ln S(I T)$ 和 $\ln S(F D I)$ 序列均 为 $I(1)$ 过程, 且变量之间在滞后期内存在稳定的协整关系, 为了进一步验证国际贸易和FDI途径下的R\&D资本存量以 及自主R\&D投入与中国TFP之间的关系, 需要就各变量分别 对TFP进行格兰杰因果关系检验。由于格兰杰因果关系检 验的关键在于滞后期的选择, 为了对不同滞后期的检验结 果进行比较, 本文同时给出了滞后期为 $2 、 3 、 4$ 的检验结 果。

通过对表 3 的分析发现，在滞后 2 期时，自主R\&D投入 是TFP提升的Grange原因; 国际贸易途径下的R\&D溢出与 TFP之间为非Granger因果关系; 而FDI途径下的R\&D溢出与 TFP之间为单项Granger因果关系, 即FDI途径下R\&D溢出是 TFP提升的Grange原因。在滞后3期时, 自主R\&D投入与TFP 之间仍为单项Granger因果关系; TFP仍不是国际贸易途径 下R\&D溢出的Grange原因, 但国际贸易途径下的R\&D溢出是 TFP的Grange原因; FDI途径下的R\&D溢出与TFP之间为双向
Granger因果关系。滞后 4 期时, 国际贸易和FDI途径下R\&D 溢出以及自主R\&D投入与TFP之间均为双向Granger因果关 系。从实证结果来看, 国际R\&D溢出与自主R\&D投入对TFP 提升的促进作用均存在时滞性。短期内, 国际R\&D溢出、 自主R\&D投入与TFP之间不存在显著的Granger因果关系; 但长期来看, 国际R\&D溢出、自主R\&D投入对TFP提升的促 进作用不断显现, 且二者之间互为Granger因果关系。

表3 各变量间的Granger因果关系检验。

\begin{tabular}{llll}
\hline 变量 & 零假设 & 滞后期 & $\begin{array}{l}\text { 检验 } \\
\text { 结论 }\end{array}$ \\
\hline \multirow{3}{*}{$\Delta \ln S(O)$} & $\Delta \ln T F P$ 不是 $\Delta \ln S(O)$ 的格兰杰原因 & 2 & 接受 \\
& $\Delta \ln S(O)$ 不是 $\Delta \ln T F P$ 的格兰杰原因 & & 拒绝 \\
$\Delta \ln S(O)$ & $\Delta \ln T F P$ 不是 $\Delta \ln S(O)$ 的格兰杰原因 & 3 & 接受 \\
& $\Delta \ln S(O)$ 不是 $\Delta \ln T F P$ 的格兰杰原因 & & 拒绝 \\
$\Delta \ln S(O)$ & $\Delta \ln T F P$ 不是 $\Delta \ln S(O)$ 的格兰杰原因 & 4 & 拒绝 \\
& $\Delta \ln S(O)$ 不是 $\Delta \ln T F P$ 的格兰杰原因 & & 拒绝 \\
$\Delta \ln S(I T)$ & $\Delta \ln T F P$ 不是 $\Delta \ln S(I T)$ 的格兰杰原因 & \multirow{2}{*}{ 接受 } \\
& $\Delta \ln S(I T)$ 不是 $\Delta \ln T F P$ 的格兰杰原因 & & 接受 \\
$\Delta \ln S(I T)$ & $\Delta \ln T F P$ 不是 $\Delta \ln S(I T)$ 的格兰杰原因 & 3 & 接受 \\
& $\Delta \ln S(I T)$ 不是 $\Delta \ln T F P$ 的格兰杰原因 & & 拒绝 \\
$\Delta \ln S(I T)$ & $\Delta \ln T F P$ 不是 $\Delta \ln S(I T)$ 的格兰杰原因 & & 拒绝 \\
& $\Delta \ln S(I T)$ 不是 $\Delta \ln T F P$ 的格兰杰原因 & 4 & 拒绝 \\
$\Delta \ln S(F D I)$ & $\Delta \ln T F P$ 不是 $\Delta \ln S(F D I)$ 的格兰杰原因 & & 接受 \\
& $\Delta \ln S(F D I)$ 不是 $\Delta \ln T F P$ 的格兰杰原因 & 2 & 拒绝 \\
& $\Delta \ln T F P$ 不是 $\Delta \ln S(F D I)$ 的格兰杰原因 & & 拒绝 \\
$\Delta \ln S(F D I)$ & $\Delta \ln S(F D I)$ 不是 $\Delta \ln T F P$ 的格兰杰原因 & 3 & 拒绝 \\
& $\Delta \ln T F P$ 不是 $\Delta \ln S(F D I)$ 的格兰杰原因 & & 拒绝 \\
& $\Delta \ln S(F D I)$ 不是 $\Delta \ln T F P$ 的格兰杰原因 & 4 & 拒绝 \\
\hline
\end{tabular}

\section{5. 结论}

本文基于永续盘存法估算出具有物量和价格双重属 性的资本服务物量指数, 采用迭代比例拟合算法将就业人 数及其劳动报酬按行业、性别、年龄、文化程度进行四维 交叉分类测算出中国劳动投入指数, 在增长核算框架下对 中国全要素生产率进行更为谨慎的再测算。在此基础上, 采用1985-2010年时间序列样本数据, 运用ADF单位根检验、 协整检验和Granger因果关系检验, 从国际贸易、FDI两个 主要溢出途径实证研究R\&D溢出与中国生产率增长的相关 关系。经验分析表明: 中国TFP的提高取决于国内自主R\&D 投入和国际R\&D溢出的综合效益, 其中自主R\&D投入在推动 TFP提高的过程中起主要作用, 一国研发投入强度的提高 是一国经济增长的根本。国际贸易和FDI途径下的R\&D溢出 对中国TFP的推动作用具有一定的时滞效应, 且在短期内 两者之间的因果关系不明显, 长期内呈双向因果关系。

本文经验分析所蕴涵的启示是: 在保持国民经济可持 续增长的过程中, 必然要求从战略高度审视技术进步问题, 把技术进步和生产率提高作为经济增长的基本优先目标。 由于中国生产率提升是国内自主研发投入以及国际R\&D溢 出等多因素综合作用的结果, 因此在选择经济发展路径时, 首先, 应加强本国的科技投入, 创造宽松的投资和贸易政 策环境, 增强本国的自主研发能力。其次, 在国际贸易方 面, 应以引进包括人力资本在内的各种有利于自主创新的 资源为着眼点, 积极扩大技术含量较高产品的进口, 进一 
步强化进口对国内 TFP的促进作用，实现经济的可持续增 长。再次, 在FDI引进方面, 应由 “量” 向 “质” 转变, 重点转向境外欧美日大型跨国企业, 并采取优惠政策鼓励 其将自己的研发总部或分部迁移至中国, 利用境外人力资 本和创新资源来实现中国技术进步。最后, 应注重促进外 资企业与国内企业的产业关联度, 加强国内企业与外资企 业在技术研发、产品创新等方面的前向关联。

\section{致谢}

本文为国家社科基金西部项目《我国经济全要素生产 率时空演变及优化路径研究》（13XJL004）的阶段性成果 之一, 并获得中国重庆市研究生科研创新项目 (CYB15001) 的资助。

\section{参考文献}

１１）李平, 钱利. 进口贸易与外商直接投资的技术溢出效应一一 对中国各地区技术进步的实证研究 [J]. 财贸研 究, 2005(6) : 40-45。
[2] 张勇, 古明明. 再谈中国技术进步的特殊性一一中国体现式 技术进步的重估 $[\mathrm{J}]$. 数量经济技术经济研 究, 2013(8) : 3-19。

[3] 曹跃群, 秦增强, 齐倩. 中国资本服务估算 $[\mathrm{J}]$. 统计研 究, 2012 (12) : 45-52。

４4］曹跃群, 秦增强, 齐倩. 中国省际资本服务测量: 概念、框架 和指数构建 $[\mathrm{J}]$. 数量经济技术经济研究, 2013 (12) : 35-50。

［5］吴明娥, 曾国平, 曹跃群. 资源环境约束下基于资本服务的 全要素生产率增长研究 $[\mathrm{J}]$. 中国人口・资源与环 境, 2015, 25(5) : 83-91。

[6] Jorgenson, D. Capital Theory and Investment Behavior [J]. American Economic Review, 1963, 53 (2) : 247-259.

[7] 任若恩, 岳希明, 郑海涛. 中国全要素生产率的行业分析与 国际比较——中国KLEMS项目 [M]. 北京: 科学出版社, 2013。

［8］岳希明, 任若恩. 测量中国经济的劳动投入：1982-2000年 [J]. 经济研究, 2008 (3) : 16-28。

[9］李宾, 曾志雄. 中国全要素生产率变动的再测算: 1978 2007 年 $[J]$. 数量经济技术经济研究, 2009 (3) : 3-15。

[10］高静, 黄繁华. 进口贸易与中国制造业全要素生产率一一基 于进口研发溢出的视角 $[\mathrm{J}]$. 世界经济研 究, 2013(11):34-41。 